\title{
Norois
}

Environnement, aménagement, société

\section{Introduction - Le capital environnemental pour penser les dynamiques socio-environnementales des espaces emblématiques}

Introduction - Analysing Socio-Environnemental Dynamics of Iconic Natural

Places through Environnemental Capital

Greta Tommasi, Frédéric Richard et Gabrielle Saumon

\section{OpenEdition}

Journals

Édition électronique

URL : http://journals.openedition.org/norois/6077

DOI : $10.4000 /$ norois.6077

ISBN : 978-2-7535-6537-1

ISSN : 1760-8546

Éditeur

Presses universitaires de Rennes

\section{Édition imprimée}

Date de publication : 2 novembre 2017

Pagination : 7-15

ISBN : 978-2-7535-6465-7

ISSN : 0029-182X

Référence électronique

Greta Tommasi, Frédéric Richard et Gabrielle Saumon, « Introduction - Le capital environnemental pour penser les dynamiques socio-environnementales des espaces emblématiques », Norois [En ligne], 243 | 2017, mis en ligne le 20 septembre 2019, consulté le 15 mars 2021. URL : http:// journals.openedition.org/norois/6077 ; DOI : https://doi.org/10.4000/norois.6077 


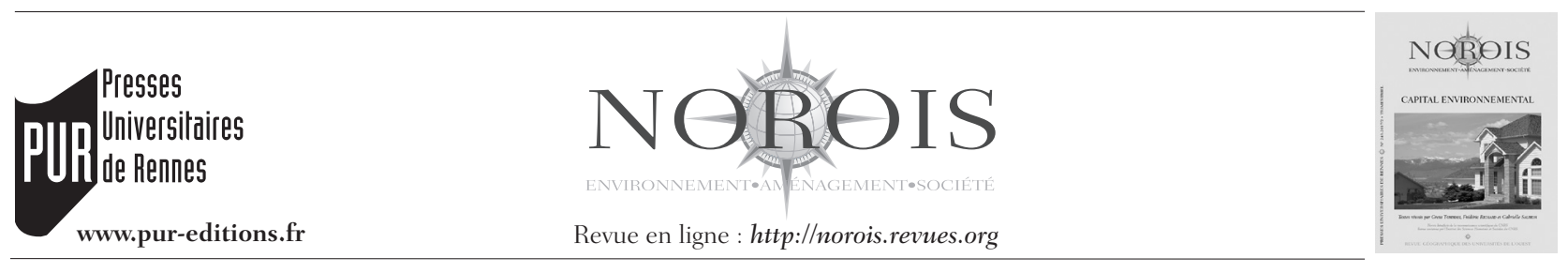

\title{
Introduction - Le capital environnemental pour penser les dynamiques socio-environnementales des espaces emblématiques
}

\author{
Introduction - Analysing Socio-Environmental Dynamics \\ of Iconic Natural Places through Environmental Capital
}

\author{
Greta Tommasi $^{\text {a }}$, Frédéric Richard ${ }^{\text {a }}$ et Gabrielle SAUmON ${ }^{a}$
}

a GEOLAB - UMR 6042 CNRS, Université de Limoges, Faculté des lettres et des sciences humaines - 39E rue

Camille-Guérin, 87036 Limoges. (frederic.richard@unilim.fr) (greta.tommasi@unilim.fr)(gabrielle.saumon@unilim.fr)

La science est en mouvement perpétuel, un mouvement animé d'expériences, d'innovations et de débats. Concernant les sciences sociales, en même temps qu'elles les observent, elles révèlent et accompagnent les sociétés dans leurs mutations. Passées au crible des questionnements scientifiques et de la vie de laboratoire (Latour, Woolgar, 1979), ces mutations nourrissent tout autant de résultats empiriques que de nouvelles réflexions et propositions conceptuelles. Le numéro 243 de Norois consacré au capital environnemental relève précisément de cette démarche consistant à formuler et tester une notion, voire un concept susceptible de rendre compte des dynamiques sociales, politiques et territoriales à l'œuvre, ici autour de la question environnementale.

Très concrètement, les premiers questionnements théoriques relatifs au capital environnemental ont été esquissés il y a quelques années au sein d'une équipe de l'UMR GEOLAB 6042 CNRS - universités de Limoges et Clermont-Auvergne dont les chercheurs s'intéressent aux interactions environnements-sociétés. Plus précisément, c'est en constatant la composante sociale et politique des percep- tions, appropriations et usages de l'environnement et/ou de la nature dans divers contextes de faibles densités, donc sur la base de travaux en cours et de premières observations empiriques, qu'a été formulée de manière à la fois très intuitive et inductive, l'hypothèse d'un capital environnemental. Celle-ci a rapidement révélé un fort potentiel à générer de l'intérêt et du débat scientifiques; le besoin et l'envie d'élargir le théâtre de ces échanges ont alors conduit à l'organisation d'un colloque à Limoges à l'automne 2015. Intitulé «Capital environnemental : représentations, pratiques, dominations, appropriations spatiales ", celui-ci a réuni une cinquantaine de communicants. Originaires de divers pays, issus d'horizons disciplinaires variés (géographie, sciences politiques, climatologie, sociologie, économie), travaillant sur des objets et des terrains également très différents les uns des autres, les chercheurs présents ont tous été interpelés et attirés par l'invitation contenue dans l'appel à communication et consistant à réfléchir, voire à construire, collectivement cette notion de capital environnemental. 


\section{DU PARADIGME ENVIRONNEMENTAL AU CAPITAL ENVIRONNEMENTAL}

Outre sa composante pluri ou interdisciplinaire, c'est a priori son potentiel opérationnel qui semble avoir facilité et stimulé les contributions. En effet, le capital environnemental était d'emblée présenté comme une grille d'analyse, un outil destiné à mieux comprendre les dynamiques socio-environnementales et découlant en partie d'une acception large, très englobante et de type plutôt constructiviste de l'environnement. Dans une perspective très proche de celle d'A. Berque (2010), celui-ci était ainsi proposé comme une construction sociale et politique, l'interaction entre une matérialité biophysique et les représentations dont elle fait l'objet, mais dont elle peut également être la projection. Au-delà de notions antérieurement privilégiées, telles que la nature ou le paysage, l'environnement comporte une dimension performative (Depraz, 2008) qui implique des considérations et des ambitions plus tangibles et opérationnelles. Il nous semblait qu'à ce titre, l'environnement était un objet passé, en quelques décennies seulement, du statut de notion émergente dans les années 1960-1970, à celui de paradigme omniprésent au sein de l'espace public (Keucheyan, 2014), pénétrant également les sciences sociales, dont la géographie (Chartier, Rodary, 2016).

De notre point de vue, cette montée en puissance de la question écologique et plus largement environnementale, en y intégrant résolument ses composantes sociale et politique, ainsi que sa visibilité croissante, elle-même liée à la fluidité de sa circulation (Latour, 2001), traduit et/ou implique l'émergence d'une nouvelle forme de capital au sein de la société. De manière très générique, le capital environnemental pourrait être défini comme l'ensemble des investissements (socio-économiques, idéologiques, émotionnels, politiques, artistiques...) dans l'environnement réalisés par des acteurs selon leurs représentations, intérêts et systèmes de valeurs spécifiques. L'investissement dans le capital environnemental se ferait au nom de valeurs du même nom, construites socialement, variées et parfois contradictoires. C'est cette proposition, mettant en jeu les différentes définitions et conceptions de l'environnement portées par les acteurs (en tant que ressource, milieu, terrain de jeu, paysage, patrimoine naturel, cadre de vie, projet politique, etc.), qui a été soumise aux réactions, critiques et débats à l'occasion du colloque et des publications qui en ont découlé.

La seule certitude affirmée d'emblée consistait à souligner que le capital environnemental se distingue très clairement du capital naturel, d'ailleurs parfois qualifié d'environnemental, en particulier dans la littérature anglophone ou nord-américaine (Gagnon et al., 2006). Le capital naturel, tel que Costanza et al. (1997) l'ont imaginé et popularisé, se nourrit d'une approche positiviste et repose sur l'évaluation du potentiel écologique et économique des éléments naturels et des processus écosystémiques qui en dépendent. Le capital environnemental s'inscrit en revanche dans une approche constructiviste et son objectif n'est pas une évaluation économique de l'environnement, mais la compréhension de dynamiques sociales (conflits, alliances, appropriations, dominations) autour de l'environnement, par le prisme des positionnements, choix et stratégies des acteurs sociaux au sein du champ environnemental mécaniquement associé au capital du même nom (cf. infra).

\section{Proposer une nouvelle grille DE LECTURE EN SCIENCES SOCIALES : ENJEUX D'UNE CIRCULATION INTER ET INFRA-DISCIPLINAIRE}

Le colloque, par la pluridisciplinarité et la diversité thématique des communications présentées, a montré la portée heuristique du capital environnemental : les chercheurs s'en sont saisis non seulement d'un point de vue théorique pour questionner les valeurs, les représentations, les processus de justice/injustice environnementale, mais surtout en tant qu'outil méthodologique qui, dans leurs terrains d'étude respectifs, permettait d'éclairer les pratiques et les interactions entre les acteurs, d'interroger des processus de protection, de valorisation ou de construction territoriale. Sa mobilisation a posteriori sur des terrains ou cas d'études déjà explorés à travers d'autres prismes, a par ailleurs montré son apport tant pour l'analyse des interactions entre les acteurs et l'environnement que dans la déconstruction des dynamiques socio-environnementales. Toutefois, si la pluridisciplinarité des contributions au colloque a confirmé la pertinence de la grille d'analyse du capital environnemental, 
le dialogue entre disciplines a présenté quelques écueils, notamment en ce qui concerne la circulation de divers concepts d'une discipline à une autre. Les échanges ont ainsi mis en lumière différentes conceptions ou acceptions disciplinaires de certains termes, mais également des divergences infra-disciplinaires, y compris entre géographes.

D'un point de vue interdisciplinaire, c'est le concept de capital qui a d'abord été questionné, en raison des différentes significations qui lui sont attribuées. En effet, imaginé dans une perspective bourdieusienne, il consiste en un potentiel dont les individus et les groupes sont plus ou moins pourvus et dont l'inégale répartition au sein de la société explique les distinctions et, le cas échéant, les inégalités entre groupes sociaux. Le champ sémantique auquel il est combiné (champs, valeurs, distribution, domination, inégalités) conforte ainsi une inscription dans la géographie critique, permettant de dévoiler et déconstruire la manière dont l'environnement alimente ou détermine des rapports de domination et parfois des processus d'exclusion. Bien qu'elles puissent leur être utiles ${ }^{1}$, ces considérations sont majoritairement absentes des sciences économiques au sein desquelles, qu'il s'agisse du capital naturel, immobilisé, humain ou total, le capital reste étroitement lié à l'idée de mesure économique. À cet égard, le colloque a rapidement posé les limites de l'usage de la notion de capital environnemental pour les économistes, certains pointant le risque de conforter une vision marchande de l'environnement.

Pour les chercheurs en sociologie en revanche, alors que l'approche bourdieusienne des capitaux peut sembler plus évidente, c'est l'introduction de nouveaux capitaux qui peut être objet de réticences. La prise de position d'Erik Neveu (2013) à cet égard est très claire : l'auteur affirme que la structuration bourdieusienne des capitaux en " trois plus un ${ }^{2}$ » suffit pour rendre compte de la complexité sociale et des inégalités entre les acteurs. Or, l'inflation actuelle de nouvelles formes de capital révèle selon Neveu un " appauvrissement conceptuel » (ibid., p. 352), d'autant plus que leur définition reste parfois incomplète et leurs relations avec les autres capitaux ne sont pas éclaircies. Pour autant, il recon-

1. Par exemple pour reconnaitre et mesurer des éléments intangibles, liés aux perceptions ou aux pratiques sociales dans le cadre des services écosystémiques.

2. Le capital culturel, économique, social, plus le symbolique qui peut être considéré en tant que «sublimation » des précédents. nait le fait que l'introduction d'un nouveau capital puisse être pertinente et utile, s'il est considéré comme un « combinatoire » ou une variante des « espèces fondatrices » (ibid., p. 354).

Les divergences autour des acceptions du capital ne se trouvent pas seulement dans le dialogue entre disciplines, mais également dans une perspective infra-disciplinaire : les géographes ont en effet eu recours au concept de capital et l'ont appliqué à leurs objets d'étude, de manière plus ou moins orthodoxe vis-à-vis des travaux d'origine de Bourdieu. Il a par exemple été question de capital « territorial » (Desponds, 2008; Lacquement, Quéva, 2016), « paysager» (Cailly et Dodier, 2007) ou encore de « countryside capital» (Garrod et al., 2006) : le concept est alors associé $\mathrm{au}(\mathrm{x})$ territoire(s), et tend à caractériser un ensemble de ressources (naturelles, sociales) favorisant leur développement et/ou attractivité. En l'occurrence, en affectant aux territoires la capacité à " détenir » un capital, cette approche s'éloigne de la pensée de Bourdieu, selon laquelle ce sont les individus et les groupes sociaux qui en disposent. D'autres géographes à l'inverse adoptent cette dernière approche centrée sur les acteurs, pour souligner le rôle de l'espace ou de sa maîtrise dans leurs trajectoires sociales et résidentielles. Il a alors été question de « capital spatial » (Lévy et al., 1993), « résidentiel » (Cailly, 2007) ou encore « géographique » (Lazzarotti, 2006), qui viennent d'une certaine manière combler la quasi-absence de la composante spatiale dans l'approche proposée par Bourdieu. Ces applications du capital sont néanmoins questionnées, notamment par Ripoll (2013; Rippol, Veschambre, 2005), pour qui la dimension spatiale chez Bourdieu, tout en étant discrète, n'est pas pour autant absente. En effet, les capitaux s'inscrivent dans des espaces, où ils sont accumulés, mobilisés, et leur potentiel varie d'un espace ou d'un territoire à l'autre. En conséquence, et justement en raison de sa dimension transversale, l'espace ne peut pas être considéré comme un capital en soi, car il ne peut pas être identifié en tant que champ autonome $^{3}$ : l'espace devrait donc être considéré en tant que dimension présente dans toute forme de capital (Ripoll, 2013). De fait, dans le capital environnemental, ce n'est pas l'espace qui représente un capital, mais bien l'environnement, considéré en

3. Dans la pensée de Bourdieu, tout capital présuppose l'existence d'un champ correspondant. 
tant que potentiel valorisable par les acteurs sociaux et dont les inégales possibilités d'accès, de pratique, d'utilisation, déterminent des rapports de force et de domination entre acteurs.

À cet égard, et toujours dans une perspective infra-disciplinaire, le colloque a amené à interroger les articulations entre capital environnemental et d'autres concepts. Plus particulièrement, comme le montrent les contributions au présent numéro, la ressource territoriale, telle que théorisée par Gumuchian et Pecqueur (2007), présenterait des passerelles avec le capital environnemental. Les deux concepts font en effet référence à un potentiel, construit sur des composantes matérielles et idéelles, que des acteurs sociaux activent. Cependant, la ressource territoriale est une notion économique et géographique qui permet de comprendre l'émergence et la construction de projets de développement localisés. Il s'agit d'un potentiel qui, par une intentionnalité politique et des synergies entre acteurs sociaux et économiques propres à chaque territoire, est activé dans une logique de développement et d'aménagement. Pour sa part, le capital environnemental ne vise pas à identifier des leviers de développement local (qui peuvent néanmoins émerger de manière indirecte) mais, par une analyse des pratiques, des stratégies, des investissements des individus et groupes sociaux dans l'environnement, à comprendre les (nouvelles) formes d'inégalités et de domination liées à celui-ci.

Ainsi, au regard de ces questionnements interet infra-disciplinaires, comment se positionner? Faudrait-il entériner "un moratoire sur l'invention de nouveaux capitaux tant qu'une discussion sérieuse n'a pas eu lieu quant aux conditions de légitimité » de leur introduction (Neveu, 2013)? Par-delà, comment situer cette grille de lecture au sein de la géographie?

En la matière, le colloque, tout comme les contributions à ce numéro, ont été l'occasion de préciser le cadre dans lequel le capital environnemental s'inscrit, à savoir celui de la géographie sociale en général, et pas exclusivement relative aux questions environnementales. La géographie sociale est ici entendue comme sous-champs disciplinaire mettant l'accent « sur les interactions de rapports sociaux et spatiaux, sur les relations entre dynamiques sociales et spatiales et sur les formes géographiques, notamment inégalitaires ou ségrégatives, à la fois maté- rielles et idéelles, concrètes et symboliques qui résultent de ces intersections et superpositions actives de rapports »(Di Meo, 2008). Dans cette perspective, le capital environnemental, en tant qu'outil de la géographie sociale, vise à envisager la manière dont l'environnement peut constituer une clé de lecture des relations sociales, notamment dans des contextes spatiaux sous tensions, tels que peuvent l'être des espaces qui, à divers titres, apparaissent comme emblématiques.

\section{Capital environnemental ET ESPACES EMBLEMATIQUES}

En l'occurrence, ce numéro spécial tend à approfondir une lecture du capital environnemental attentive au processus de construction de valeurs environnementales normées et/ou dominantes sur des espaces emblématiques. À travers les articles retenus, il s'agit d'interroger l'apport de cette grille de lecture à la démarche géographique, le capital environnemental s'inscrivant de fait dans la discipline puisqu'il appelle une analyse située, propre à un contexte territorial donné et auquel correspond donc un champ environnemental spécifique (cf. infra). Mais plus singulièrement, le choix de consacrer ce numéro à la relation entre capital environnemental et espaces emblématiques repose sur la capacité de ces derniers à concentrer les enjeux socio-environnementaux : espaces caractérisés par de fortes aménités ou par leur capacité à incarner des valeurs environnementales, ils sont au coeur des stratégies de protection et/ou de valorisation d'acteurs conscients de leurs potentialités, et cristallisent parfois les tensions. Dans son article sur les Plus Beaux Villages de France, Hélène Ducros fait ainsi référence au patrimoine architectural et naturel reconnu et protégé de villages dont le caractère rural nourrit une identité elle-même fondée sur l'esthétique paysagère. L'article de Véronique AndréLamat, Mayté Banzo et Solange Pupier-Dauchez interroge la construction de valeurs dans la dune du Pilat, haut lieu touristique et labélisé, objet de pratiques et représentations variées de la part de touristes, habitants et acteurs économiques et institutionnels. Frédéric Richard, Greta Tommasi et Gabrielle Saumon soulignent le rôle des aménités environnementales dans le processus de gentrification rurale observé en Angleterre, dans le Montana 
et dans la Montagne Limousine, espaces emblématiques d'une nature fantasmée ou convoitée (rural idyll, wilderness et campagne alternative). Myriam Simard s'appuie elle aussi sur un contexte rural en proie à la gentrification rurale, considéré dans son ensemble comme emblématique au regard des qualités environnementales et paysagères qu'il est censé incarner. Cependant, en explorant plus en détail des objets et lieux de controverses environnementales, elle propose en complément une vision très localisé de l'espace emblématique. Valerian Geffroy et Samuel Depraz proposent un article sur les Biosphärenparks autrichiens, territoires modèles et consensuels en cela qu'ils tendent à répondre aux objectifs du développement durable. Emblématiques d'une attention particulière portée à l'environnement, ces espaces peuvent d'ailleurs faire l'objet d'une protection sous la forme d'une labellisation : Biosphärenparks de l'Unesco (Geffroy, Depraz), Plus Beaux Villages de France (Ducros), Grand Site de France (André-Lamat, Banzo, PupierDauchez), parcs nationaux ou régionaux et Area of Outstanding Natural Beauty (Richard, Tommasi, Saumon). Simon Maraud et Caroline Desbiens posent en revanche la question des interprétations de l'emblématique : la Baie James incarne, à différentes échelles et pour différents acteurs, les grands enjeux politico-économiques de la confrontation entre représentations de la nature occidentales et cris. Si le caractère emblématique des espaces étudiés semble faire consensus dans les quatre autres articles de ce numéro, il est pour autant au fondement des concurrences entre les groupes dans la Baie James, c'est-à-dire dans un contexte géographique très spécifique car héritier direct de la période coloniale (Maraud, Desbiens).

\section{Des dynamiques portées par des valeurs environnementales}

La place de l'environnement dans les dynamiques des espaces étudiés a ainsi invité les auteurs du numéro à interroger la reconnaissance des enjeux environnementaux dans les sociétés, dans un contexte de crise écologique. La formalisation d'un cadre de pensée normatif autour de l'environnement est d'ailleurs rendue manifeste par le renouvellement des approches scientifiques, notamment dans une perspective holiste. Les acteurs sociaux sont en effet désormais amenés à se positionner au regard de valeurs environnementales légitimées et légitimantes, ce qui nous invite à envisager l'émergence d'un champ environnemental, condition préalable à l'existence d'un capital environnemental. En empruntant à Bourdieu sa terminologie et sa démonstration, le champ environnemental peut être défini comme l'espace social structuré autour de l'environnement et au sein duquel les acteurs se réfèrent à des valeurs environnementales selon leurs intérêts propres (Bourdieu, 2000). Dans les espaces emblématiques étudiés, les acteurs interagissent et prennent part à des luttes sociales afin de se positionner dans ce champ de forces, au nom des valeurs qu'ils défendent, et expriment celles-ci par des opérations concrètes sur l'espace que le géographe peut analyser. S'appuyant sur une typologie déjà formalisée (Richard, 2017), Frédéric Richard, Greta Tommasi et Gabrielle Saumon soulignent, dans les dynamiques qui animent les gentrifieurs, l'importance des valeurs liées à l'habitabilité (authenticité architecturale, esthétique domestique, inscription dans le paysage...), à la qualité environnementale et aux pratiques écologisantes (éco-responsabilité, respect des ressources via les pratiques et engagements), aux composantes productive (agriculture, foresterie) et enfin post-productive de l'environnement (l'espace rural pensé alors comme lieu de récréation ou d'agrément). L'article de Véronique André-Lamat, Mayté Banzo et Solange PupierDauchez s'intéresse au processus de construction de la valeur (environnementale, mais également économique et sociale) : les auteures soulignent le paradoxe entre un espace qui, tout en ayant une faible valeur économique, fait l'objet d'intérêt et d'investissements différenciés selon les acteurs sociaux. Ceux-ci (touristes, habitants, acteurs économiques ou pouvoirs publics) investissent la dune au nom de valeurs socio-environnementales, qu'elles soient individuelles (l'expérience physique et émotionnelle liée à la pratique de la dune, les intérêts économiques) ou collectives (associées au processus de patrimonialisation), et qui peuvent être à l'origine d'interactions conflictuelles. Les valeurs peuvent également être liées à la tradition et au régionalisme : elles se manifestent ainsi à travers la labellisation des produits du Biosphärenpark présentés comme locaux, naturels et traditionnels, exprimant la « qualité du lieu » (Geffroy, Depraz). Il s'agit dans ce der- 
nier cas de souligner spécifiquement la mobilisation et la diffusion de ces valeurs environnementales "sous la forme d'éléments de communication, de discours (verbaux, visuels ou matériels) qui mettent en correspondance ces valeurs avec des objets du territoire " (Geffroy, Depraz). De la même manière, Hélène Ducros interroge le processus de définition de valeurs environnementales mis en œuvre par la Charte de qualité de l'association des Plus Beaux Villages de France. Identifiant vingt-sept critères, relatifs au patrimoine naturel, urbanistique et architectural notamment, nécessaires pour obtenir le label, elle produit et véhicule « des valeurs multiples mais précises, [et] se transforme en recueil de valeurs, délimitant ce champ de l'environnement » (Ducros). Dans ces deux articles, les auteurs interrogent ainsi la labellisation comme formalisation de valeurs environnementales. Si cette dernière apparait bien consensuelle dans ces deux cas précis, tout l'enjeu dans la Baie James réside au contraire dans l'opposition entre des valeurs occidentales liées à la composante productive de l'environnement d'un côté (ressources naturelles à exploiter dans cadre de l'économie de marché) et des valeurs cris de l'autre, liées à l'autochtonie et, dans un deuxième moment, à l'écologie (Maraud, Desbiens). Du reste, cette dichotomie entre des valeurs, d'un côté associées à la protection de l'environnement et des paysages pour les néo-ruraux, et de l'autre justifiant au contraire une vision productiviste et financière pour les entrepreneurs et élus locaux, traverse également les analyses de M. Simard.

\section{Investir et convertir : des opérations stratégiques sur l'environnement}

Mentionner la conflictualité manifeste entre les acteurs de la Baie James nous invite, après avoir démontré la place des valeurs environnementales dans les dynamiques étudiées, à interroger la façon dont le capital environnemental peut constituer une grille de lecture opérante. Les stratégies de protection ou de valorisation des espaces emblématiques par les différents acteurs reposent en effet sur leur investissement dans l'environnement, selon les valeurs qui les animent et qu'ils défendent. Cet investissement recouvre divers procédés selon les contextes étudiés : dans le cadre des Biosphärenparks, il relève de stratégies de commu- nication de la part d'acteurs locaux conscients des potentialités de leur territoire, dont ils présentent les produits et pratiques en convoquant une rhétorique du naturel, du traditionnel et du local, mais également de projets d'aménagements et de pratiques de l'espace qui viennent conforter une vision partagée de l'espace emblématique (Geffroy, Depraz). De la même manière, afin d'obtenir le label des Plus Beaux Villages de France, les élus locaux investissent dans l'environnement en s'appuyant sur les valeurs environnementales définies par la charte, ce qui renforce l'esprit du lieu et revitalise les villages (Ducros). Autour de la dune du Pilat, une conflictualité est susceptible d'émerger entre des acteurs sociaux qui investissent dans cet espace au nom de valeurs hétérogènes : l'expérience et la pratique pour les touristes, la qualité écologique et la protection pour les institutions, l'habitabilité pour les habitants, l'attractivité pour les acteurs touristiques (André-Lamat, Banzo, Pupier-Dauchez). Dans les campagnes gentrifiées d'Angleterre, du Montana et de la Montagne Limousine, les nouveaux-arrivants investissent dans leur habitat par des rénovations ou des constructions neuves, par le recours aux matériaux locaux ou écologiques, avec une attention manifeste portée aux paysages - donc selon leurs valeurs environnementales spécifiques. Mais bien au-delà de l'habitat, les gentrifieurs investissent dans l'environnement au travers de sa dimension postproductive, via des pratiques récréatives et de pleine nature (Richard, Tommasi, Saumon), y compris au Québec (Simard).

Pour investir dans l'environnement, les acteurs convertissent leurs capitaux déjà acquis en capital environnemental - cette opération de conversion venant d'ailleurs légitimer l'existence d'un capital environnemental autonome, plutôt qu'en tant que combinatoire des autres capitaux (cf. partie précédente). Ainsi, en achetant des propriétés de luxe dans un cadre paysager particulièrement attractif, ou encore en finançant la protection de l'environnement via des opérations de mécénat, les gentrifieurs convertissent leur capital économique en capital environnemental. Parce qu'ils disposent également de capital social et de capital culturel, ils s'appuient sur leurs réseaux pour réaliser des projets répondant à leurs valeurs environnementales et sont en mesure d'influencer les politiques d'urbanisme ou d'aménagement (Richard, Tommasi, Saumon), voire de pro- 
tection de l'environnement. C'est le cas notamment à l'occasion de la controverse étudiée par M. Simard autour d'un projet de carrière porté par des élus et entrepreneurs et finalement annulé à l'issue d'une accélération des opérations de conversion de tous types de capitaux (culturels, sociaux, politiques) en faveur du capital environnemental. Véronique André-Lamat, Maïté Banzo et Solange PupierDauchez décrivent les processus d'expropriation et de rachat engagés par des acteurs publics (Syndicat Mixte en partenariat avec le Conservatoire du Littoral), manifestes d'une forme de conversion de capital économique et symbolique en capital environnemental, au nom des enjeux de préservation et de patrimonialisation. Mais si l'investissement dans le capital environnemental se fait par un jeu de conversions, il peut également être convertible à court ou long terme en d'autres formes de capitaux, selon les intérêts et les stratégies des acteurs. Ainsi les Cris, considérés par les auteurs comme des nouveaux entrants dans le champ de l'environnement, investissent aujourd'hui non seulement dans la composante productive, mais également dans la qualité paysagère, la qualité écologique et la pratique récréative. Ils tendent ainsi à convertir leur capital environnemental hérité en capital économique, en favorisant l'attractivité touristique de leur territoire dorénavant protégé, tout en maintenant leur système de valeurs également hérité (Maraud, Desbiens). Ces opérations de conversion peuvent aussi être le fait de stratégies collectives afin de valoriser un territoire : ainsi, la labellisation des Biosphärenparks en fait des territoires modèles en termes de développement durable, convertissant ainsi le capital environnemental en capital symbolique collectif (Geffroy, Depraz). Dans le cas des Plus Beaux Villages de France, l'investissement des acteurs locaux dans le capital environnemental est immédiatement converti en capital économique (nouvelles opportunités de développement touristique et plus généralement territorial), social (en rejoignant le réseau national et international des Plus Beaux Villages de France) et surtout symbolique : la rhétorique identitaire nouvellement investie valorise alors l'imaginaire collectif de la «villagité », faisant du village un emblème de la ruralité (Ducros).

\section{UNE NOUVELLE APPROCHE DES INJUSTICES ENVIRONNEMENTALES?}

Dans ces espaces emblématiques qui concentrent les enjeux environnementaux, les stratégies d'acteurs révèlent parfois des valeurs antagonistes, et les investissements réalisés dans l'environnement peuvent cristalliser les tensions. C'est notamment le cas dans la Baie James, où le rapport de force entre gouvernement et Nation crie est manifeste dans un premier temps, pour ensuite laisser place à une logique de co-construction du capital environnemental. Or, la coopération qui s'ensuit est à interroger comme acceptation d'un cadre de pensée occidentalo-normé par une Nation crie partagée entre ses valeurs écologisantes et leur interprétation économique imposée depuis le mouvement de colonisation (Maraud et Desbiens). La dune du Pilat est également un lieu qui cristallise les tensions autour des usages de cet espace, et la conciliation entre logiques de protection, de sécurisation et de publicisation peut être complexe. Les tensions sont aussi manifestes entre des usagers dont les pratiques sont considérées comme plus ou moins légitimes : c'est le cas par exemple des controverses opposant des touristes pratiquant des sports élitistes (parapente) et des vacanciers ayant un usage également récréatif de la dune, mais plus conventionnel et grand public (André-Lamat, Banzo, Pupier-Dauchez). Les investissements dans l'environnement semblent alors souvent réalisés dans des cadres particulièrement normatifs, au nom de valeurs socialement reconnues et légitimées. L'équilibre entre les valeurs du champ environnemental est ainsi orchestré par des producteurs symboliques, qui formalisent, diffusent voire imposent des valeurs dominantes. Valérian Geffroy et Samuel Depraz reconnaissent ainsi dans les Biosphärenparks une «situation relativement consensuelle », qui relève d'« un cadre idéologique bien identifié, celui du développement durable». Ce cadre de pensée prescrit de manière non coercitive une interprétation légitime de l'environnement, et les acteurs locaux se prêtent d'autant plus facilement au jeu de la labellisation qu'elle répond, de manière plus ou moins consciente, à une représentation partagée car finalement imposée. En l'espèce, Hélène Ducros interroge au contraire la formalisation, par le label des Plus Beaux Villages de France, d'un ensemble de valeurs environne- 
mentales qui ne sont pas toujours partagées par les habitants, symboliquement dépossédés du lieu par sa mise en marque. Dans ce processus de standardisation de la spécificité rurale, est finalement imposé un système de valeurs élitiste et normatif par des élus locaux soucieux d'obéir aux exigences d'une labellisation prescrivant la juste définition de l'entité villageoise. En témoigne la mise à l'écart des valeurs ne remplissant pas les critères définis par la Charte - les valeurs écologiques notamment, les panneaux solaires, jugés inesthétiques, étant rejetés... Si le contexte des campagnes québécoises diffère quelque peu, les néo-ruraux fondent eux aussi leurs conceptions récréatives et protectionnistes de l'environnement et des paysages sur des valeurs légitimes et légitimées dans d'autres sphères (à l'échelle provinciale ou nationale par exemple) et parviennent à les imposer (au moins parfois) au sein de territoires où elles sont pourtant moins soutenues par les acteurs locaux, posant la question de la marginalisation de ces derniers (Simard).

Finalement, la grille de lecture par le capital environnemental permet de mettre en lumière les rapports de force entre les acteurs du champ environnemental, plus ou moins aptes à formaliser et diffuser leur système de valeurs. Mais au-delà, elle tend à révéler un nouvel actif social qui, inégalement réparti et maîtrisé, entretient voire aggrave les inégalités entre les acteurs : leur inégale capacité d'investissement dans le capital environnemental permet d'interroger la (re)production d'une logique dominants/dominés dans l'interaction société-environnement. Comme toutes les autres espèces de capitaux, le capital environnemental est en effet inégalement distribué. Les acteurs, pour disposer de capital environnemental, investissent dans des biens, du savoir-faire et du savoir être, selon leurs valeurs environnementales, et en convertissant leurs capitaux préexistants : ce jeu de conversion d'une forme de capital en une autre explique en partie la hiérarchie au sein du champ environnemental. Ainsi, en Angleterre, dans le Montana et dans la Montagne limousine, les gentrifieurs investissent dans l'environnement au nom de valeurs qu'ils portent et diffusent, voire imposent, parce qu'ils disposent déjà de capitaux économiques, sociaux et culturels. Par là, ils affirment, ou confirment leur position sociale au sein de ces territoires et contribuent à des processus d'exclusion symbolique et/ou matérielle des populations plus modestes ou qui ne partageraient pas les mêmes valeurs (Richard, Tommasi, Saumon). Le rapport de domination peut également évoluer dans le temps, comme le montrent Maraud et Desbiens : le rapport de force entre gouvernent québécois et Nation crie a été pendant longtemps en faveur du premier, qui disposait du monopole de production et légitimation des valeurs environnementales. Il pouvait ainsi déterminer les formes de développement et de planification du territoire, excluant les Cris de toute décision sur leur territoire de vie. La diffusion de nouvelles valeurs et de nouvelles formes de conversion a ensuite permis de rééquilibrer le rapport de force entre gouvernement québécois et autochtones. Cette perspective diachronique permet de souligner les évolutions, voire les fluctuations du capital environnemental au gré de l'émergence et/ou de l'affaiblissement relatif de certaines des valeurs constitutives du champ environnemental, qui peut accompagner l'affirmation de nouvelles formes et relations de pouvoirs sur les territoires.

Ainsi, en éclairant les rapports de force et les inégalités liées à l'environnement, le capital environnemental peut enrichir la notion de justice/injustice environnementale : encore en construction au sein de la géographie sociale en France, celle-ci est plurielle et difficile à conceptualiser, tant les échelles, les acteurs, les formes d'injustices et les conceptions de l'environnement sont variées (Blanchon et al., 2009). La grille d'analyse par le capital environnemental permet d'éclairer le processus de construction des injustices, par le prisme des valeurs environnementales, normatives et exclusives, que les groupes sociaux dominants produisent et s'approprient. Elle permet alors d'envisager inégalités sociales et iniquités environnementales et inégalités non seulement du côté des dominés, mais peut-être plus encore, de celui des dominants.

\section{Bibliographie}

Berque A., 2010, Histoire de l'habitat idéal: De l'Orient vers l'Occident, Paris, Éd. du Félin, 396 p.

Blanchon D., Moreau S., Veyret Y., 2009. Comprendre et construire la justice environnementale, Annales de géographie, vol. 1, no 665-666, p. 35-60.

Bourdieu P., 2000. Propos sur le champ politique, Paris, Presses universitaires de Lyon, $112 \mathrm{p}$. 
Cailly L., 2007. Capital spatial, stratégies résidentielles et processus d'individualisation, Annales de géographie vol. 2, $n^{\circ} 654$, p. 169-187.

Cailly L., Dodier R., 2007. La diversité des modes d'habiter des espaces périurbains dans les villes intermédiaires : différenciations sociales, démographiques et de genre, Norois. Environnement, aménagement, société, 205, p. 67-80.

Chartier D., Rodary E. (dir.), 2016. Manifeste pour une géographie environnementale, Paris, Presses de Sciences Po, 412 p.

Costanza R., D’Arge R., de Groot R., Farber S., Grasso M., Hannon B., 1997. The value of the world's ecosystem services and natural capital, Nature 387, p. 253-260.

Depraz S., 2008. Géographie des espaces naturels protégés. Genèse, principes et enjeux territoriaux, Paris, Armand Colin, $320 \mathrm{p}$.

Desponds D., 2008. Contribution des démarches prospectives différenciées au renforcement des logiques ségrégatives. Exploration dans l'aire d'influence de la «Ville nouvelle » de Cergy-Pontoise, in Séchet R., Garat I., Zeneidi D. (dir.), Espaces en transaction, Rennes, PUR, p. 47-66.

Di Meo G., Une géographie sociale entre représentations et action, Montagnes méditerranéennes et développement territorial, 2008, p. 1-9, [https://halshs.archives-ouvertes.fr/ halshs-00281573].

Gagnon C., Simard J.-G., Tellier L.N., Gagnon S., 2008, Développement territorial viable, capital social et capital environnemental : quels liens?, Vertigo. La revue électronique en sciences de l'environnement, 8, 2, [http://vertigo. revues.org/4983].

Garrod B., Wornell R., Youell R., 2006. Re-conceptualising rural resources as countryside capital: The case of rural tourism, Journal of Rural Studies 22, 1, p. 117-128.
Gumuchian H., Pecqueur B., 2007. La ressource territoriale, Paris, Economica, 252 p.

Keucheyan R., 2014. La nature est un champ de bataille, Paris, La Découverte, 176 p.

Lacquement G., Queva C., 2016. Introduction. Innovations sociales et développement des territoires dans les campagnes européennes. Norois, n 241, 4, p. 7-13.

Latour B., Woolgar S., 2006 [1979]. La vie de laboratoire. La production des faits scientifiques, Paris, La Découverte, 299 p.

Latour B., 2007 [2001]. L'espoir de Pandore : pour une version réaliste de l'activité scientifique, Paris, La Découverte, 347 p.

Lazzarotti O., 2006. Habiter, aperçus d'une science géographique, Cahiers de géographie du Québec 50, 139, p. 85-102.

Lévy J., Déloye Y., Haegel F., 1993. Espace intime, espace légitime? Paris, l'urbain, l'urbanité, Politix 6, 21, p. 52-64.

Neveu É., 2013. Les sciences sociales doivent-elles accumuler les capitaux?, Revue française de science politique, 63, 2, p. 337-358.

Richard F., 2017. La gentrification rurale, de l'observation du fait géographique à la circulation du concept, Habilitation à diriger des recherces, vol. 1, Université de Limoges, 227 p.

Ripoll F., 2013. Quelle dimension spatiale des structures sociales chez Bourdieu? Localisations résidentielles et jeux d'échelles dans La Distinction, in Coulangeon Ph., Duval J. (dir.), Trente ans après La Distinction, Paris, La Découverte, p. 365-377.

Ripoll F., Veschambre V., 2005. Sur la dimension spatiale des inégalités : contribution aux débats sur la «mobilité et le capital spatial », in Arlaud S., Jean Y., Rououx D. (dir.), Rural-Urbain. Nouveaux liens, nouvelles frontières, Rennes, PUR, p. 467-483. 